\title{
MÉTODO DOS MÍNIMOS QUADRADOS PONDERADOS NA ANÁLISE DE GRUPOS DE EXPERIMENTOS
}

\author{
Wilson Alves de Oliveira ${ }^{1}$, Maria Cristina Stolf Nogueira ${ }^{2}$
}

${ }^{1}$ Doutorando em Estatística e Experimentação Agronômica - ESALQ/USP. Av. Pádua Dias, 11 Cx. Postal 9 - CEP-
$13418-900$ - Piracicaba -SP.
${ }^{2}$ Prof..Drª. Universidade de São Paulo - Depto de Ciências Exatas/ESALQ. Av. Pádua Dias, 11 Cx. Postal 9 - CEP-
$13418-900$ - Piracicaba -SP.

\section{RESUMO}

A análise de grupos de experimentos apresenta alguns problemas como heterogeneidade de variâncias dos diferentes experimentos, número de repetições diferentes, para tratamentos, em cada local, heterogeneidade das variâncias das interações e outros fatores. Neste trabalho é utilizado o método dos mínimos quadrados ponderados para contornar o problema de heterogeneidade de variâncias dos diferentes experimentos. Os dados são referentes a um grupo de 11 experimentos com cana de açúcar, apresentado por Malavolta et al. (1963).

Palavras-chave: Análise de variância, interação, heterogeneidade de variâncias.

\section{ABSTRACT}

\section{WEIGHTED LEAST SQUARES METHOD IN THE ANALYSIS OF GROUPS OF EXPERIMENTS}

The analysis of groups of experiments presents some problems as heterogeneity of variances from place to place, different number of replications to treatments in each place, heterogeneity of the interactions variances and other factors. This work presents the weighted least squares method to solve the problem of heterogeneity of variances from place to place. The data refer a group of 11 experiments with sugar cane presented by Malavolta et al. (1963).

Key words: Analysis of variance, interaction, heterogeneity of variances.

\section{INTRODUÇÃO}

$\mathrm{Na}$ experimentação agronômica,
algumas vezes, existe o interesse do
pesquisador em obter conclusões gerais para
um conjunto de experimentos. Estes
experimentos podem ser conduzidos em
vários locais distintos e/ou em várias épocas
diferentes. Pode haver várias razões para
isto, como obter informações que possam ser
aplicadas a uma população que é extensiva
seja em empaco e/ou em tempo. Em geral, o objetivo da pesquisa é produzir resultados que possam ser aplicados a toda população.

A metodologia estatística aplicada para realizar uma análise estatística para os dados de uma série de experimentos irá variar com o objetivo da pesquisa. Em geral, o objetivo é verificar se os tratamentos, aplicados em vários locais ou em diferentes épocas, produzem efeitos diferentes. Além disso, é necessário que a homocedasticidade, das variâncias residuais entre experimentos, esteja presente para que a aplicação, dos 
métodos estatísticos na análise conjunta, não leve a conclusões errôneas. Assim, o pesquisador deve estar atento para as dificuldades que surgem em uma análise conjunta.

$\mathrm{Na}$ análise estatística conjunta, Cochran (1937) obteve as estimativas dos efeitos de tratamentos, e as correspondentes estimativas dos erros padrão. $\mathrm{O}$ autor considerou, ainda, que se as estimativas puderem ser consideradas igualmente precisas, a análise de variância é facilmente obtida. No caso contrário, sugere utilizar a média ponderada, como uma estimativa apropriada da média, quando ocorrer pelo menos 15 graus de liberdade associados à estimativa da variância do resíduo.

Um exame cuidadoso dos dados, antes de iniciar qualquer análise conjunta foi sugerido por Yates \& Cochran (1938). Os autores indicam que o procedimento de análise de variância usual, apropriado para análises de um único experimento, pode necessitar de modificações, devido a falta de homogeneidade das variâncias dos resíduos entre os experimentos e, também, à presença da heterocedasticidade nos componentes da interação de tratamentos e locais. Recomendam o uso da análise da variância ponderada para contornar estes problemas.

$\mathrm{Na}$ análise conjunta Kempthorne (1952) considera a existência de duas possíveis dificuldades para a interpretação da análise de variância. A primeira é que a variância não é constante para os experimentos e a segunda dificuldade é que a variância da interação de tratamentos e locais depende da combinação de tratamentos e locais, ou seja, em um local o material experimental pode não responder a todos os tratamentos, enquanto em outro pode haver uma pequena resposta para alguns tratamentos e uma grande resposta para outros.

Um método que consiste em fazer um ajuste nos números de graus de liberdade, para a interação e para o resíduo médio, quando os quadrados médios dos resíduos para cada experimento são muito diferentes, foi proposto por Cochran (1954). Na presença de heterocedasticidade de variâncias, o teste $\mathrm{F}$ para interações é aproximado, assim a distribuição $\mathrm{F}$ ainda poderia ser usada como uma aproximação, fazendo-se uma correção no número de graus de liberdade. Porém, há uma objeção de que o $\mathrm{F}$ pode ser insensível em detectar a presença de interações, pois experimentos com baixa precisão recebem o mesmo peso que aqueles com maior precisão. Como alternativa, o autor sugere basear o teste de interações na soma de quadrados dos desvios ponderados.

Ao se realizar a análise preliminar de dados para a análise conjunta, duas críticas foram feitas por Cochran \& Cox (1957). A primeira é que alguns quadrados médios estimados para os efeitos da interação experimentos e tratamentos podem ser muito diferentes entre si, ou seja, a variância da interação não é constante. A segunda crítica é a presença da heterocedasticidade entre experimentos. Segundo os autores, isto pode invalidar o teste $\mathrm{F}$ aplicado aos efeitos de interesse, produzindo resultados distorcidos. Sugerem, como alternativa, o uso da análise de variância ponderada.

Os experimentos podem ser reunidos e analisados conjuntamente sem problema, de acordo com Pimentel Gomes (1990), desde que os quadrados médios residuais não difiram muito entre si, isto é, que sejam relativamente homogêneos. Sugere o teste de Bartlett para verificação da homogeneidade de variâncias, ressaltando que este teste é sensível à falta de normalidade.

O objetivo deste trabalho é contornar o problema de heterogeneidade das variâncias entre os experimentos, quando da realização da análise conjunta, aplicando o método dos Mínimos Quadrados Ponderados (Cochran \& Cox, 1957). 


\section{MATERIAL E MÉTODOS}

Com o objetivo de apresentar a aplicação do Método dos Mínimos Quadrados Ponderados na análise conjunta de dados experimentais de um grupo de experimentos, com variâncias heterocedásticas, considerou-se os dados apresentados em Malavolta et al. (1963), referentes a rendimentos de cana-de-açúcar, $\mathrm{em}$ t/ha, de 11 experimentos instalados em terra roxa legítima e em locais diferentes. Cada experimento foi instalado seguindo o esquema fatorial $3^{3}$, envolvendo 3 doses eqüidistantes para $\mathrm{N}, \mathrm{P}$ e $\mathrm{K}$. A técnica do confundimento de 2 graus de liberdade da interação tripla $\mathrm{N} \times \mathrm{P} \times \mathrm{K}$ com o efeito de blocos foi aplicada. Assim, cada experimento foi constituído por 3 blocos constituídos de 9 parcelas cada um, sendo que os tratamentos foram distribuídos aos blocos de acordo com o grupo de confundimento W desenvolvido por Yates ( 1937). No trabalho apresentado por Malavolta et al. (1963), para realizar a análise estatística conjunta dos experimentos, os autores agruparam os 11 experimentos em 2 subgrupos aproximadamente, homogêneos, constatado através dos Quadrados Médios Residuais das análises de variância individuais. Neste trabalho considerou-se, para a análise conjunta, os 11 experimentos.

$\mathrm{Na}$ análise de dados de grupos de experimentos deve-se, inicialmente, realizar a análise de variância de cada experimento individualmente. A finalidade seria a obtenção dos Quadrados Médios do Resíduo para o teste de homocedasticidade. $O$ modelo matemático adotado é:

$$
y_{(i) j k l m}=\mu \dashv b_{j} \dashv N_{k} \dashv P_{l} \dashv K_{m} \dashv(N P)_{k l} \dashv(N K)_{k m} \dashv(P K)_{l m} \dashv e_{(i) j k l m}
$$

onde:

$\begin{array}{ll}y_{(i) j k l m} & \text { produção de cana-planta, em t/ha, no bloco } j, \text { no local i e na } \\ & k l m \text {-ésima combinação de } N, P \text { e } K \text { respectivamente; } i=1, \ldots, I ; \\ & j=1, \ldots, J ; k=1, \ldots, K ; l=1, \ldots, L \text { e } m=1, \ldots, M ; \\ & \text { média geral; } \\ b_{j} & \text { efeito dos blocos; } \\ N_{k} & \text { efeito do fator Nitrogênio, considerado fixo; } \\ P_{l} & \text { efeito do fator Fósforo, considerado fixo; } \\ K_{m} & \text { efeito do fator Potássio, considerado fixo; } \\ (N P)_{k l} & \text { efeito da interação Nitrogênio e Fósforo, considerado fixo; } \\ (N K)_{k m} & \text { efeito da interação Nitrogênio e Potássio, considerado fixo; } \\ (P K)_{l m} & \text { efeito da interação Fósforo e Potássio, considerado fixo; } \\ e_{(i) j k l m} & \text { erro experimental, } e_{(i) j k l m} \sim \text { NID }\left(0, \sigma_{(i)}^{2}\right) .\end{array}$

No caso dos experimentos fatoriais $3^{3}$ com confundimento de 2 graus de liberdade da interação tripla com blocos e com uma única repetição, segundo alguns autores, entre eles Banzatto \& Kronka (1989), a parte não confundida da interação tripla, com 6 graus de liberdade, funciona como resíduo.
Para aumentar os graus de liberdade do resíduo, visando obter melhores conclusões, as interações duplas envolvendo $\mathrm{N}, \mathrm{P}$, e $\mathrm{K}$, com 4 graus de liberdade, podem ser desdobradas, isolando-se apenas a interação entre os componentes lineares. Desta maneira, o resíduo médio fica constituído 
pelo acréscimo dos graus de liberdade das demais interações, não consideradas como causa de variação da análise da variância conjunta, totalizando 15 graus de liberdade.

$$
\begin{aligned}
& y_{i j k l m}=\mu \dashv E_{i} \dashv b_{(i) j} \dashv N_{k} \dashv P_{l} \dashv K_{m} \dashv(N P)_{k l} \dashv(N K)_{k m} \dashv(P K)_{l m} \dashv(E N)_{i k} \dashv(E P)_{i l} \dashv \\
& \dashv(E K)_{i m} \dashv(E N P)_{i k l} \dashv(E N K)_{i k m} \dashv(E P K)_{i m} \dashv e_{i j k l m}
\end{aligned}
$$

onde: para a $k l m$-ésima combinação dos fatores $N, P$ e $K$ respectivamente;

$\mu \quad \begin{array}{ll}i=1, \ldots, \mathrm{I} ; j \\ \quad \text { média geral; }\end{array}$

$E_{i} \quad$ efeito do experimento $i$, considerado aleatório;

$b_{(i) j} \quad$ efeito dos blocos dentro do experimento $i$, considerado fixo;

$N_{k} \quad$ efeito do fator Nitrogênio, considerado fixo;

$P_{l} \quad$ efeito do fator Fósforo, considerado fixo;

$K_{m} \quad$ efeito do fator Potássio, considerado fixo;

$(N P)_{k l} \quad$ efeito da interação Nitrogênio e Fósforo, considerado fixo;

$(N K)_{k m}$ efeito da interação Nitrogênio e Potássio, considerado fixo;

$(P K)_{l m}$ efeito da interação Fósforo e Potássio, considerado fixo;

$(E N)_{i k}$ efeito da interação Experimento e Nitrogênio, considerado aleatório;

$(E P)_{i l}$ efeito da interação Experimento e Fósforo, considerado aleatório;

$(E K)_{i m}$ efeito da interação Experimento e Potássio, considerado aleatório;

$(E N P)_{i k l}$ efeito da interação Experimento, $N$ e $P$, considerado aleatório;

$(E N K)_{i k m}$ efeito da interação Experimento, $N e K$, considerado aleatório;

$(E P K)_{i l m}$ efeito da interação Experimento, $P$ e K, considerado aleatório;

$e_{i j k l m} \quad$ erro aleatório, normal e independentemente distribuído, com média zero e variância $c^{2}$.

A metodologia a ser aplicada na análise dos dados que seguem o modelo adotado irá depender da presença ou ausência da homocedasticidade entre os experimentos. Se for constatada a presença da heterocedasticidade, uma das alternativas apropriadas é a aplicação do Método dos Mínimos Quadrados Ponderados.

\section{Método dos Mínimos Quadrados Ponderados}

$\mathrm{Na}$ presença da heterogeneidade de variâncias entre os experimentos, segundo Cochran \& Cox (1957), o teste F para interações, tendo por denominador $\mathrm{o}$ quadrado médio do resíduo médio, não é válido. Embora o efeito sobre o nível de significância de $F$ não seja conhecido exatamente, ele opera de forma que se tenham muitos resultados significativos onde não existiriam. A alternativa mais simples para isso, segundo os autores, é o uso da análise de variância ponderada.

Quando se usa a análise ponderada, a soma de quadrados dos resíduos ponderados $\sum_{i} w_{i}\left(x_{i}-\bar{x}_{w}\right)^{2}$ é minimizada, sendo $w_{i}=1 / s_{i}^{2}$, onde $s_{i}^{2}$ é a variância estimada por experimento, $x_{i}$ é o valor observado da 
variável resposta e $\bar{x}_{w}$ é a média ponderada dada por

$$
\bar{x}_{w}=\frac{\sum_{i=1}^{I} w_{i} x_{i}}{w}
$$

onde, $\quad w=\sum_{i} w_{i}$ e $I$ é o número de experimentos.

O uso da ponderação $r_{i} / s_{i}^{2}$, onde $r_{i}$ é o número de repetições por experimento, é mais indicado que a ponderação $1 / s_{i}^{2}$, quando se têm diferentes números de repetições nos experimentos.

As equações normais usadas na análise ponderada são: $\hat{\beta}=\left(X^{\prime} W X\right)^{-} X^{\prime} W Y$, onde $W$ é uma matriz diagonal contendo os valores das ponderações $w_{i}$.

Se as ponderações são proporcionais aos recíprocos das variâncias dos resíduos, então as estimativas de mínimos quadrados ponderados são BLUE (Best Linear Unbiased Estimators).

Para realizar as análises propostas, desenvolveu-se um programa utilizando o software SAS (Statistical Analysis System) conforme apresentado em Oliveira (1999).

\section{RESULTADOS}

Utilizando os quadrados médios dos resíduos, para cada experimento, apresentados na tabela 1 , verificou-se a hipótese de homogeneidade entre as variâncias residuais dos experimentos.

Tabela 1. Quadrados médios dos resíduos para os experimentos.

\begin{tabular}{ccc} 
Experimentos (i) & GL & QMResíduo (i) \\
\hline 1 & 15 & 118,41 \\
2 & 15 & 61,95 \\
3 & 15 & 104,58 \\
4 & 15 & 117,82 \\
5 & 15 & 280,02 \\
6 & 15 & 470,91 \\
7 & 15 & 231,96 \\
8 & 15 & 302,49 \\
9 & 15 & 608,83 \\
10 & 15 & 250,25 \\
11 & 15 & 500,36 \\
\hline
\end{tabular}

Os testes utilizados foram de Hartley (Milliken \& Johnson, 1992) e o de Bartlett (Snedecor \& Cochran, 1967). A hipótese de homogeneidade foi rejeitada, ao nível de 5\% de probabilidade.

Os resultados da análise de variância ponderada são apresentados na tabela 2 . 
Tabela 2. Análise de variância ponderada.

\begin{tabular}{|c|c|c|c|c|c|}
\hline Causas de Variação & G.L. & S.Q. & Q.M. & $\mathrm{F}$ & $\operatorname{Pr}>\mathrm{F}$ \\
\hline Experimentos (E) & 10 & 78,7081 & 7,8708 & 1473,27 & $<0,0001$ \\
\hline Blocos d. E & 22 & 0,2980 & 0,0135 & - & - \\
\hline $\mathrm{N}$ & 2 & 0,2363 & 0,1182 & 3,91 & 0,0369 \\
\hline$P$ & 2 & 0,0267 & 0,0149 & 1,94 & 0,1698 \\
\hline $\mathrm{K}$ & 2 & 0,5690 & 0,2845 & 7,63 & 0,0034 \\
\hline $\mathrm{N}^{\prime} \times \mathrm{P}^{\prime}$ & 1 & 0,0002 & 0,0002 & 0,03 & 0,8659 \\
\hline$N^{\prime} \times K^{\prime}$ & 1 & 0,0016 & 0,0016 & 0,31 & 0,5899 \\
\hline $\mathrm{P}^{\prime} \times \mathrm{K}^{\prime}$ & 1 & 0,0005 & 0,0005 & 0,13 & 0,7259 \\
\hline $\mathrm{E} \times \mathrm{N}$ & 20 & 0,6046 & 0,0302 & 5,12 & 0,0393 \\
\hline $\mathrm{E} \times \mathrm{P}$ & 20 & 0,1538 & 0,0077 & 1,64 & 0,3397 \\
\hline $\mathrm{E} \times \mathrm{K}$ & 20 & 0,7451 & 0,0373 & 10,97 & 0,0359 \\
\hline $\mathrm{E} \times \mathrm{N}^{\prime} \times \mathrm{P}^{\prime}$ & 10 & 0,0653 & 0,0065 & 1,12 & 0,3501 \\
\hline $\mathrm{E} \times \mathrm{N}^{\prime} \times \mathrm{K}^{\prime}$ & 10 & 0,0520 & 0,0052 & 0,90 & 0,5346 \\
\hline $\mathrm{E} \times \mathrm{P}^{\prime} \times \mathrm{K}^{\prime}$ & 10 & 0,0396 & 0,0040 & 0,69 & 0,7328 \\
\hline Resíduo Médio & 165 & 0,9506 & 0,0058 & - & - \\
\hline Total & 296 & 82,4544 & - & & \\
\hline
\end{tabular}

\section{DISCUSSÃO}

Pela tabela 2, verifica-se que os efeitos de $\mathrm{E} \times \mathrm{N}$ e $\mathrm{E} \times \mathrm{K}$ foram significativos $(p=0,05)$, indicando que os efeitos do nitrogênio $(\mathrm{N})$ e do potássio $(\mathrm{K})$ têm um comportamento diferenciado dentro de cada experimento. Deve-se, então, estudar estes efeitos, isoladamente, dentro de cada local.

As interações de primeira ordem, $\mathrm{N}^{\prime} \times \mathrm{P}^{\prime}, \mathrm{N}^{\prime} \times \mathrm{K}^{\prime}$ e $\mathrm{P}^{\prime} \times \mathrm{K}^{\prime}$, foram testadas com os respectivos resíduos específicos, $\mathrm{E} \times \mathrm{N}^{\prime} \times \mathrm{P}^{\prime}, \mathrm{E} \times \mathrm{N}^{\prime} \times \mathrm{K}^{\prime}$ e $\mathrm{E} \times \mathrm{P}^{\prime} \times \mathrm{K}^{\prime} . \mathrm{O}$ uso do resíduo específico teve como objetivo contornar o problema da heterogeneidade de variâncias das interações. Os resíduos específicos foram obtidos através da decomposição das soma de quadrados de tratamentos e da soma de quadrados das interações em um conjunto de componentes ortogonais.

\section{CONCLUSÃO}

O uso do método dos mínimos quadrados ponderados é uma alternativa para o caso de heterogeneidade entre as variâncias residuais dos experimentos, evitando assim a divisão dos experimentos em subgrupos homogêneos, procedimento que foge da característica inicial da pesquisa.

\section{REFERÊNCIAS BIBLIOGRÁFICAS}

BANZATTO, D. A., KRONKA, S. N. (1989). Experimentação agrícola. Jaboticabal: FUNEP-UNESP, 247p.

COCHRAN, W. G. (1937). Problems arising in the analysis of a series of similar experiments. J. R. Stat. Soc., v.4, p.102-118.

COCHRAN, W. G. (1954). The combination of estimates from different experiments.

Biometrics, v.10, p.101-129.

COCHRAN, W. G., COX, G. M. (1957). Experimental Designs. 2.ed. New York: John Wiley and Sons, 611p. 
KEMPTHORNE, O. (1952). The design and analysis of experiments. New York: John Wiley and Sons, 631p.

MALAVOLTA, E. et al. (1963). A diagnose foliar na cana-de-açúcar IV. Resultados de 40 ensaios fatoriais NPK $3 \times 3 \times 3$, primeiro corte no estado de São Paulo. Piracicaba: ESALQ-USP, 47p.

MILLIKEN, G. A., JOHNSON, D. E. (1992). Analysis of messy data. v.1. New York: Chapman \& Hall, 473p.

OLIVEIRA, W. A. (1999). Análise de grupos de experimentos com heterogeneidade de variâncias. (Dissertação de Mestrado) - Escola Superior de Agricultura "Luíz de
Queiroz"/Universidade de São Paulo, Piracicaba, 97p.

PIMENTEL GOMES, F. (1990). Curso de estatística experimental. 13. ed. Piracicaba: Nobel, 477p.

SAS Institute Inc., SAS/STAT ${ }^{\mathrm{TM}}$ (2006). User's Guide, Release 9.1.ed. Cary, NC: SAS Institute Inc..

SNEDECOR, G. W., COCHRAN, W. G. (1967). Statistical Methods. 2.ed. Iowa: The Iowa State University Press, 593 p.

YATES, F. (1937). The design and analysis of factorial experiments. Imp. Bureau Soil Sci., v.35.

YATES, F., COCHRAN, W. G. (1938). The analysis of groups of experiments. $\mathbf{J}$. Agric. Sci., v.28, p.556-580. 